\title{
Discovery of VHE $\gamma$-rays from the high-frequency-peaked BL Lacertae object RGB J0152+017
}

F. Aharonian ${ }^{1,13}$, A. G. Akhperjanian² ${ }^{2}$ U. Barres de Almeida ${ }^{8, \star}$, A. R. Bazer-Bachi ${ }^{3}$, B. Behera ${ }^{14}$, M. Beilicke $^{4}$, W. Benbow ${ }^{1}$, K. Bernlöhr, ${ }^{1,5}$, C. Boisson ${ }^{6}$, V. Borrel ${ }^{3}$, I. Braun ${ }^{1}$, E. Brion ${ }^{7}$, J. Brucker ${ }^{16}$, R. Bühler ${ }^{1}$, T. Bulik ${ }^{24}$, I. Büsching ${ }^{9}$, T. Boutelier ${ }^{17}$, S. Carrigan ${ }^{1}$, P. M. Chadwick ${ }^{8}$, R. C. G. Chaves ${ }^{1}$, L.-M. Chounet ${ }^{10}$, A. C. Clapson ${ }^{1}$, G. Coignet ${ }^{11}$, R. Cornils ${ }^{4}$, L. Costamante ${ }^{1,28}$, M. Dalton ${ }^{5}$, B. Degrange ${ }^{10}$, H. J. Dickinson ${ }^{8}$, A. Djannati-Ataï ${ }^{12}$, W. Domainko ${ }^{1}$, L. O.'C. Drury ${ }^{13}$, F. Dubois ${ }^{11}$, G. Dubus ${ }^{17}$, J. Dyks ${ }^{24}$, K. Egberts ${ }^{1}$, D. Emmanoulopoulos ${ }^{14}$, P. Espigat ${ }^{12}$, C. Farnier ${ }^{15}$, F. Feinstein ${ }^{15}$, A. Fiasson ${ }^{15}$, A. Förster ${ }^{1}$, G. Fontaine ${ }^{10}$, M. Füßling ${ }^{5}$, S. Gabici ${ }^{13}$, Y. A. Gallant ${ }^{15}$, B. Giebels ${ }^{10}$, J.-F. Glicenstein ${ }^{7}$, B. Glück ${ }^{16}$, P. Goret ${ }^{7}$, C. Hadjichristidis ${ }^{8}$, D. Hauser ${ }^{14}$, M. Hauser ${ }^{14}$, G. Heinzelmann ${ }^{4}$, G. Henri ${ }^{17}$, G. Hermann ${ }^{1}$, J. A. Hinton ${ }^{25}$, A. Hoffmann ${ }^{18}$, W. Hofmann ${ }^{1}$, M. Holleran ${ }^{9}$, S. Hoppe ${ }^{1}$, D. Horns ${ }^{4}$, A. Jacholkowska ${ }^{15}$, O. C. de Jager ${ }^{9}$, I. Jung ${ }^{16}$, K. Katarzyński ${ }^{27}$, S. Kaufmann ${ }^{14}$, E. Kendziorra ${ }^{18}$, M. Kerschhaggl ${ }^{5}$, D. Khangulyan ${ }^{1}$, B. Khélifi ${ }^{10}$, D. Keogh ${ }^{8}$, Nu. Komin ${ }^{15}$, K. Kosack ${ }^{1}$, G. Lamanna ${ }^{11}$, I. J. Latham ${ }^{8}$, J.-P. Lenain 6 , T. Lohse ${ }^{5}$, J.-M. Martin ${ }^{6}$, O. Martineau-Huynh ${ }^{19}$, A. Marcowith ${ }^{15}$, C. Masterson ${ }^{13}$, D. Maurin ${ }^{19}$,

T. J. L. McComb ${ }^{8}$, R. Moderski ${ }^{24}$, E. Moulin ${ }^{7}$, M. Naumann-Godo ${ }^{10}$, M. de Naurois ${ }^{19}$, D. Nedbal ${ }^{20}$, D. Nekrassov $^{1}$, S. J. Nolan ${ }^{8}$, S. Ohm ${ }^{1}$, J.-P. Olive ${ }^{3}$, E. de Oña Wilhelmi ${ }^{12}$, K. J. Orford ${ }^{8}$, J. L. Osborne ${ }^{8}$, M. Ostrowski ${ }^{23}$, M. Panter1', G. Pedaletti ${ }^{14}$, G. Pelletier ${ }^{17}$, P.-O. Petrucci ${ }^{17}$, S. Pita ${ }^{12}$, G. Pühlhofer ${ }^{14}$, M. Punch ${ }^{12}$, A. Quirrenbach ${ }^{14}$,

B. C. Raubenheimer ${ }^{9}$, M. Raue ${ }^{1}$, S. M. Rayner ${ }^{8}$, M. Renaud ${ }^{1}$, F. Rieger ${ }^{1}$, J. Ripken $^{4}$, L. Rob ${ }^{20}$, S. Rosier-Lees $^{11}$, G. Rowell ${ }^{26}$, B. Rudak ${ }^{24}$, J. Ruppel ${ }^{21}$, V. Sahakian ${ }^{2}$, A. Santangelo ${ }^{18}$, R. Schlickeiser ${ }^{21}$, F. M. Schöck ${ }^{16}$, R. Schröder ${ }^{21}$, U. Schwanke ${ }^{5}$, S. Schwarzburg ${ }^{18}$, S. Schwemmer ${ }^{14}$, A. Shalchi ${ }^{21}$, H. Sol ${ }^{6}$, D. Spangler ${ }^{8}$, Ł. Stawarz $^{23}$,

R. Steenkamp ${ }^{22}$, C. Stegmann ${ }^{16}$, G. Superina ${ }^{10}$, P. H. Tam ${ }^{14}$, J.-P. Tavernet ${ }^{19}$, R. Terrier ${ }^{12}$, C. van Eldik ${ }^{1}$, G. Vasileiadis ${ }^{15}$, C. Venter ${ }^{9}$, J.-P. Vialle ${ }^{11}$, P. Vincent ${ }^{19}$, M. Vivier ${ }^{7}$, H. J. Völk ${ }^{1}$, F. Volpe ${ }^{10,28}$, S. J. Wagner ${ }^{14}$, M. Ward ${ }^{8}$, A. A. Zdziarski ${ }^{24}$, and A. Zech ${ }^{6}$

(Affiliations can be found after the references)

Received 18 February 2008 / Accepted 26 February 2008

\section{ABSTRACT}

Aims. The BLLac object RGB J0152+017 ( $z=0.080)$ was predicted to be a very high-energy (VHE; $>100 \mathrm{GeV}) \gamma$-ray source, due to its high $\mathrm{X}$-ray and radio fluxes. Our aim is to understand the radiative processes by investigating the observed emission and its production mechanism using the High Energy Stereoscopic System (HESS) experiment.

Methods. We report recent observations of the BL Lac source RGB J0152+017 made in late October and November 2007 with the HESS array consisting of four imaging atmospheric Cherenkov telescopes. Contemporaneous observations were made in X-rays by the Swift and RXTE satellites, in the optical band with the ATOM telescope, and in the radio band with the Nançay Radio Telescope.

Results. A signal of $173 \gamma$-ray photons corresponding to a statistical significance of $6.6 \sigma$ was found in the data. The energy spectrum of the source can be described by a powerlaw with a spectral index of $\Gamma=2.95 \pm 0.36_{\text {stat }} \pm 0.20_{\text {syst }}$. The integral flux above $300 \mathrm{GeV}$ corresponds to $\sim 2 \%$ of the flux of the Crab nebula. The source spectral energy distribution (SED) can be described using a two-component non-thermal synchrotron self-Compton (SSC) leptonic model, except in the optical band, which is dominated by a thermal host galaxy component. The parameters that are found are very close to those found in similar SSC studies in TeV blazars.

Conclusions. RGB J0152+017 is discovered as a source of VHE $\gamma$-rays by HESS The location of its synchrotron peak, as derived from the SED in Swift data, allows clear classification as a high-frequency-peaked BL Lac (HBL).

Key words. galaxies: BL Lacertae objects: individual: RGB J0152+017 - gamma rays: observations - galaxies: BL Lacertae objects: general galaxies: active

\section{Introduction}

First detected as a radio source (Becker et al. 1991) by the NRAO Green Bank Telescope and in the Parkes-MIT-NRAO surveys (Griffith et al. 1995), RGB J0152+017 was later identified as a BL Lac object by Laurent-Muehleisen et al. (1998),

^ Supported by CAPES Foundation, Ministry of Education of Brazil. who located it at $z=0.080$, and was claimed as an intermediatefrequency-peaked BL Lac object by Laurent-Muehleisen et al. (1999). Brinkmann et al. (1997) report the first detection of RGB J0152+017 in X-rays in the ROSAT-Green Bank (RGB) sample. The host is an elliptical galaxy with luminosity $M_{R}=$ -24.0 (Nilsson et al. 2003). The source has high radio and X-ray fluxes, making it a good candidate for VHE emission 
(Costamante \& Ghisellini 2002), motivating its observation by the HESS experiment.

The broad-band SED of BLLac objects is typically characterised by a double-peaked structure, usually attributed to synchrotron radiation in the radio-to-X-ray domain and inverse Compton scattering in the $\gamma$-ray domain, which is frequently explained by SSC models (see, e.g., Aharonian et al. 2005). However, since the flux of BL Lac objects can be highly variable (e.g. Krawczynski et al. 2000), stationary versions of these models are only relevant for contemporaneous multi-wavelength observations of a non-flaring state. The contemporaneous radio, optical, X-ray, and VHE observations presented here do not show any significant variability, and thus enable the first SSC modelling of the emission of RGB J0152+017.

\section{HESS observations and results}

RGB J0152+017 was observed by the HESS array consisting of four imaging atmospheric Cherenkov telescopes, located in the Khomas Highland, Namibia (Aharonian et al. 2006a). The observations were performed from October 30 to November 14, 2007. The data were taken in wobble mode, where the telescopes point in a direction typically at an offset of $0.5^{\circ}$ from the nominal target position (Aharonian et al. 2006a). After applying selection cuts to the data to reject periods affected by poor weather conditions and hardware problems, the total live-time used for analysis amounts to $14.7 \mathrm{~h}$. The mean zenith angle of the observations is $26.9^{\circ}$.

The data are calibrated according to Aharonian et al. (2004). Energies are reconstructed taking the effective optical efficiency evolution into account (Aharonian et al. 2006a). The separation of $\gamma$-ray-like events from cosmic-ray background events was made using the Hillas moment-analysis technique (Hillas 1985). Signal extraction was performed using standard cuts (Aharonian et al. 2006a). The on-source events were taken from a circular region around the target with a radius of $\theta=0.11^{\circ}$. The background was estimated using reflected regions (Aharonian et al. 2006a) located at the same offset from the centre of the observed field as the on-source region.

A signal of $173 \gamma$-ray events is found from the direction of RGB J0152+017. The statistical significance of the detection is $6.6 \sigma$ according to $\mathrm{Li} \& \mathrm{Ma}$ (1983). The preliminary detection was reported by Nedbal et al. (2007). A two-dimensional Gaussian fit of the excess yields a position $\alpha_{J 2000}=1^{\mathrm{h}} 52^{\mathrm{m}} 33^{\mathrm{s}} .5 \pm$ $5.3_{\text {stat }} \pm 1^{\mathrm{s}} .3_{\text {syst }}, \delta_{J 2000}=1^{\circ} 46^{\prime} 40.3 \pm 107_{\text {stat }}^{\prime \prime} \pm 20_{\text {syst }}^{\prime \prime}$. The measured position is compatible with the nominal position of RGB J0152+017 $\left(\alpha_{J 2000}=1^{\mathrm{h}} 52^{\mathrm{m}} 39.78, \delta_{J 2000}=1^{\circ} 47^{\prime} 18^{\prime \prime} .70\right)$ at the $1 \sigma$ level. Given this spatial coincidence, we identify the source of $\gamma$-rays with RGB J0152+017. The angular distribution of events coming from RGB J0152+017, shown in Fig. 1, is compatible with the expectation from the Monte Carlo simulations of a point source.

Figure 2 shows the time-averaged differential spectrum. The spectrum was derived using standard cuts with an energy threshold of $300 \mathrm{GeV}$. Another set of cuts, the spectrum cuts described in Aharonian et al. (2006b), were used to lower the energy threshold and improve the photon statistics (factor $\sim 2$ increase above the standard cuts). Both give consistent results (see inlay in Fig. 2 and caption). Because of the better statistics and energy range, we use the spectrum derived using spectrum cuts in the following. Between the threshold of $240 \mathrm{GeV}$ and $3.8 \mathrm{TeV}$, this differential spectrum is described well $\left(\chi^{2} /\right.$ d.o.f. $\left.=2.16 / 4\right)$ by a power law $\mathrm{d} N / \mathrm{d} E=\Phi_{0}(E / 1 \mathrm{TeV})^{-\Gamma}$ with a photon in$\operatorname{dex} \Gamma=2.95 \pm 0.36_{\text {stat }} \pm 0.20_{\text {syst }}$ and normalisation at $1 \mathrm{TeV}$

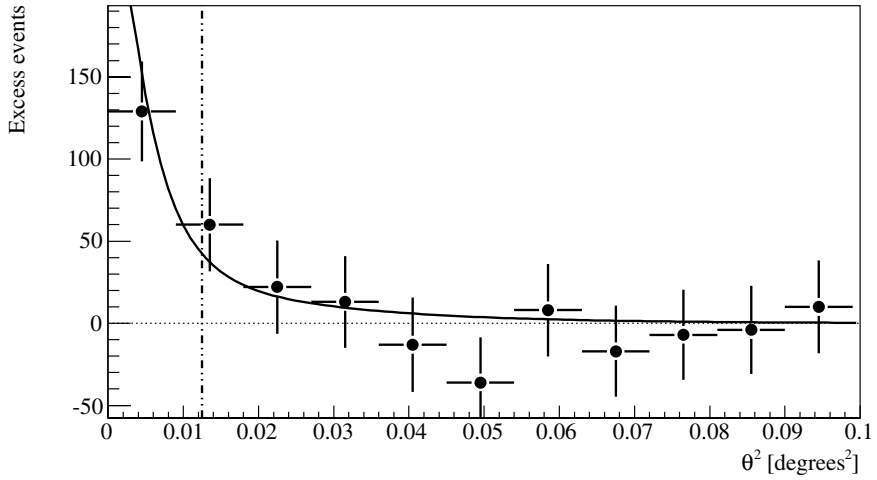

Fig. 1. Angular distribution of excess events. The dot-dashed line shows the angular distance cut used for extracting the signal. The excess distribution is consistent with the HESS point spread function as derived from Monte Carlo simulations (solid line).

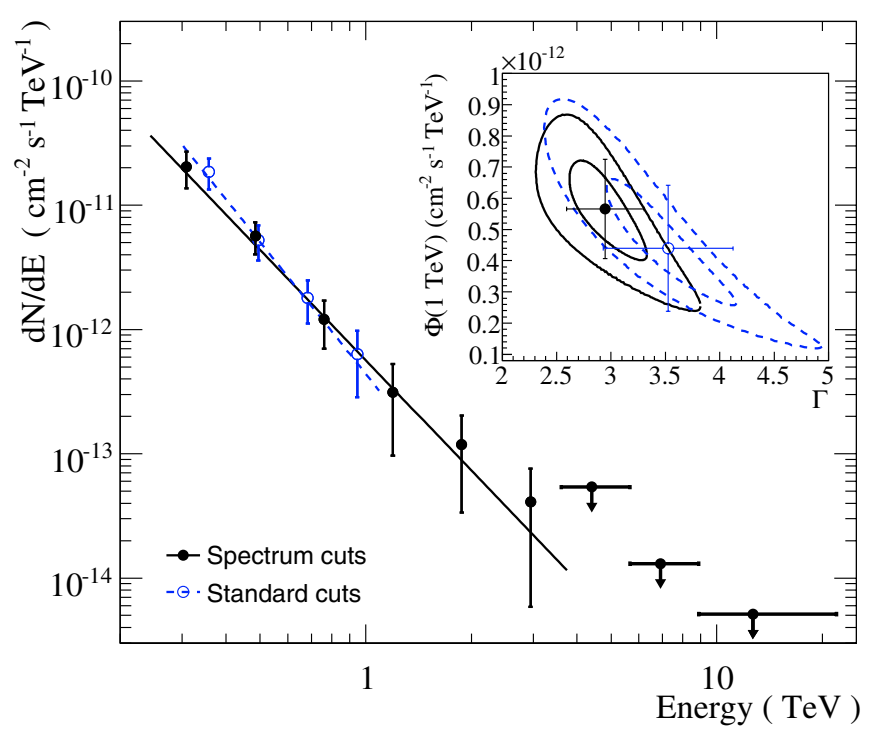

Fig. 2. Differential spectrum of RGB J0152+017. The spectrum obtained using spectrum cuts (black closed circles) is compared with the one obtained by the standard cuts (blue open circles). The black line shows the best fit by a powerlaw function of the former. The three points with the highest photon energy represent upper limits at $99 \%$ confidence level, calculated using Feldman \& Cousins (1998). All error bars are only statistical. The fit parameters of a powerlaw fit are $\Gamma=2.95 \pm 0.36_{\text {stat }} \pm 0.20_{\text {syst }}$ and $\Phi(1 \mathrm{TeV})=\left(5.7 \pm 1.6_{\text {stat }} \pm 1.1_{\text {syst }}\right) \times$ $10^{-13} \mathrm{~cm}^{-2} \mathrm{~s}^{-1} \mathrm{TeV}^{-1}$ for the spectrum cuts, and $\Gamma=3.53 \pm 0.60_{\text {stat }} \pm$ $0.2_{\text {syst }}$ and $\Phi(1 \mathrm{TeV})=(4.4 \pm 2.0) \times 10^{-13} \mathrm{~cm}^{-2} \mathrm{~s}^{-1} \mathrm{TeV}^{-1}$ for the standard cuts. The insert shows 1 and $2 \sigma$ confidence levels of the fit parameters.

of $\Phi(1 \mathrm{TeV})=\left(5.7 \pm 1.6_{\text {stat }} \pm 1.1_{\text {syst }}\right) \times 10^{-13} \mathrm{~cm}^{-2} \mathrm{~s}^{-1} \mathrm{TeV}^{-1}$. The $99 \%$ confidence level upper limits for the highest three bins shown in Fig. 2 were calculated using Feldman \& Cousins (1998).

The integral flux above $300 \mathrm{GeV}$ is $I=\left(2.70 \pm 0.51_{\text {stat }} \pm\right.$ $\left.0.54_{\text {syst }}\right) \times 10^{-12} \mathrm{~cm}^{-2} \mathrm{~s}^{-1}$, which corresponds to $\sim 2 \%$ of the flux of the Crab nebula above the same threshold as determined by Aharonian et al. (2006a). Figure 3 shows the nightly evolution of the $\gamma$-ray flux above $300 \mathrm{GeV}$. There is no significant variability between nights in the lightcurve. The $\chi^{2} /$ d.o.f. of the fit to a constant is $17.2 / 12$, corresponding to a $\chi^{2}$ probability of $14 \%$.

All results were checked with independent analysis procedures and calibration chain giving consistent results. 


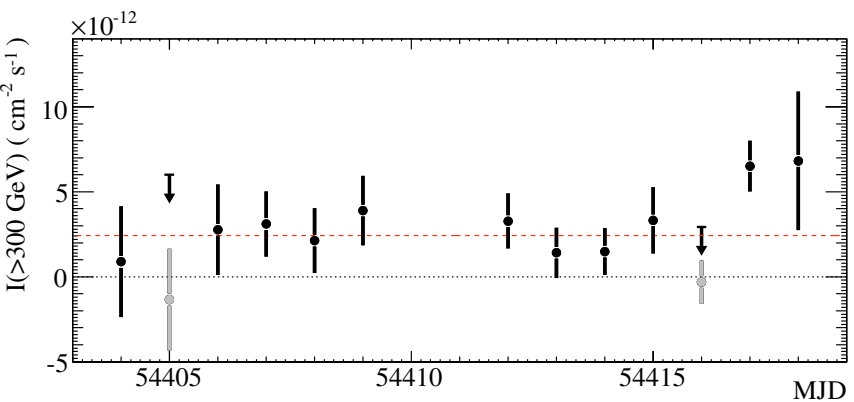

Fig. 3. Mean nightly integral flux from RGB J0152+017 above $300 \mathrm{GeV}$. Only the statistical errors are shown. Upper limits at $99 \%$ confidence level are calculated when no signal is found (grey points). The dashed line shows a fit of a constant to the data points with $\chi^{2} /$ d.o.f. of 17.2/12. The fit was performed using all nights.

\section{Multi-wavelength observations with Swift, RXTE, ATOM, and the Nançay Radio Telescope}

\subsection{X-ray data from Swift and RXTE}

Target of opportunity (ToO) observations of RGB J0152+017 were performed with Swift and RXTE on November 13, 14, and 15, 2007 triggered by the HESS discovery.

The Swift/XRT (Burrows et al. 2005) data (5.44ks) were taken in photon-counting mode. The spectra were extracted with xselect v2. 4 from a circular region with a radius of 20 pixels $\left(0.8^{\prime}\right)$ around the position of RGB J0152+017, which contains $90 \%$ of the PSF at $1.5 \mathrm{keV}$. An appropriate background was extracted from a region next to the source with four times this area. The auxiliary response files were created with the script xrtmkarf vQ.5.6 and the response matrices were taken from the Swift package of the calibration database caldb v3.4.1. Due to the low count rate of $0.3 \mathrm{cts} / \mathrm{s}$, any pileup effect on the spectrum is negligible. We find no significant variability during any of the pointings or between the three subsequent days; hence, individual spectra were combined to achieve better photon statistics. The spectral analysis was performed using the tool Xspec v11.3.2. A broken powerlaw $\left(\Gamma_{1}=1.93 \pm 0.20, \Gamma_{2}=\right.$ $2.82 \pm 0.13, E_{\text {break }}=1.29 \pm 0.12 \mathrm{keV}$ ) with a Galactic absorption of $2.72 \times 10^{20} \mathrm{~cm}^{-2}$ (LAB Survey, Kalberla et al. 2005) is a good description $\left(\chi^{2} /\right.$ d.o.f. $\left.=24 / 26\right)$, and the resulting unabsorbed flux is $F_{0.5-2 \mathrm{keV}} \sim 5.1 \times 10^{-12} \mathrm{erg} \mathrm{cm}^{-2} \mathrm{~s}^{-1}$ and $F_{2-10 \mathrm{keV}} \sim 2.7 \times 10^{-12} \mathrm{erg} \mathrm{cm}^{-2} \mathrm{~s}^{-1}$.

Simultaneous observations at higher X-ray photon energies were obtained with the RXTE/PCA (Jahoda et al. 1996). Only data of PCU2 and the top layer were taken to obtain the best signal-to-noise ratio. After filtering out the influence of the South Atlantic Anomaly, tracking offsets, and the electron contamination, an exposure of $3.2 \mathrm{ks}$ remains. Given the low count rate of $0.7 \mathrm{cts} / \mathrm{s}$, the "faint background model" provided by the RXTE Guest Observer Facility was used to generate the background spectrum with the script pcabackest v3.1. The response matrices were created with pcarsp v10.1. Again no significant variations were found between the three observations, and individual spectra were combined to achieve better photon statistics. The PCA spectrum can be described by an absorbed single powerlaw with photon index $\Gamma=2.72 \pm 0.08\left(\chi^{2} /\right.$ d.o.f. $\left.=20 / 16\right)$ between 2 and $10 \mathrm{keV}$, using the same Galactic absorption as for Swift data. The resulting flux $F_{2-10 \mathrm{keV}} \sim 6.8 \times 10^{-12} \mathrm{erg} \mathrm{cm}^{-2} \mathrm{~s}^{-1}$ exceeds the one obtained simultaneously with Swift by a factor of 2.5. We attribute this mostly to contamination by the nearby galaxy cluster Abell 267 (44.6' offset from RGB J0152+017 but still in the field of view of the non-imaging PCA).

A detailed decomposition is beyond the scope of this paper, so we exclude RXTE data from broadband modelling. The RXTE data-set confirms the absence of variability during November 2007, also in the energy range up to $10 \mathrm{keV}$. For the SED modelling, the average spectrum is treated as an upper limit. Further observations with RXTE in December 2007 also show no indication of variability.

\subsection{Optical data}

Optical observations were taken using the ATOM telescope (Hauser et al. 2004) at the HESS site from November 10, 2007. No significant variability was detected during the nights between November 10 and November 20; $R$-band fluxes binned nightly show an RMS of 0.02 mag.

Absolute flux values were found using differential photometry against stars calibrated by K. Nilsson (priv. comm.). We measured a total flux of $m_{R}=15.25 \pm 0.01$ mag (host galaxy + core) in an aperture of $4^{\prime \prime}$ radius. The host galaxy was subtracted with galaxy parameters given in Nilsson et al. (2003), and aperture correction given in Eq. (4) of Young (1976). The core flux in the $R$-band $(640 \mathrm{~nm})$ was found to be $0.62 \pm 0.08 \mathrm{mJy}$. This value was not corrected for Galactic extinction.

\subsection{Radio data}

The Nançay Radio Telescope (NRT) is a meridian transit telescope with a main spherical mirror of $300 \mathrm{~m} \times 35 \mathrm{~m}$ (Theureau et al. 2007). The low-frequency receiver, covering the band 1.8-3.5 GHz was used, with the NRT standard filterbank backend.

The NRT observations were obtained in two contiguous bands of $12.5 \mathrm{MHz}$ bandwidth, centred at 2679 and $2691 \mathrm{MHz}$ (average frequency: $2685 \mathrm{MHz}$ ). Two linear polarisation receivers were used during the 2260 -s drift scan observations on the source on November 12 and 14, 2007. The data have been processed with the standard NRT software packages NAPS and SIR. All bands and polarisations have been averaged, giving an RMS noise of $2.2 \mathrm{mJy}$. The source 3C 295 was observed for calibration, on November 11, 13, and 15, 2007.

Taking into account a flux density for this source of $12.30 \pm$ $0.06 \mathrm{Jy}$ using the spectral fit published by Ott et al. (1994), we derived a flux density of $56 \pm 6 \mathrm{mJy}$ at $2685 \mathrm{MHz}$ for RGB J0152+017. No significant variability was found in the radio data.

\section{Discussion}

Figure 4 shows the SED of RGB J0152+017 with the data from Nançay, ATOM, Swift/XRT, RXTE/PCA, and HESS. Even though some data are not strictly simultaneous, no significant variability is found in the X-ray and optical bands throughout the periods covered; hence, a common modelling of the contemporaneous X-ray and VHE data appears justified.

The optical part of the SED is mainly due to the host galaxy, which is detected and resolved in optical wavelengths (Nilsson et al. 2003). A template of the spectrum of such an elliptic galaxy is shown in the SED, as inferred from the code PEGASE (Fioc \& Rocca-Volmerange 1997). The host-galaxy-subtracted data point from the ATOM telescope might include several additional contributions - from an accretion disk, an extended jet (see below), 


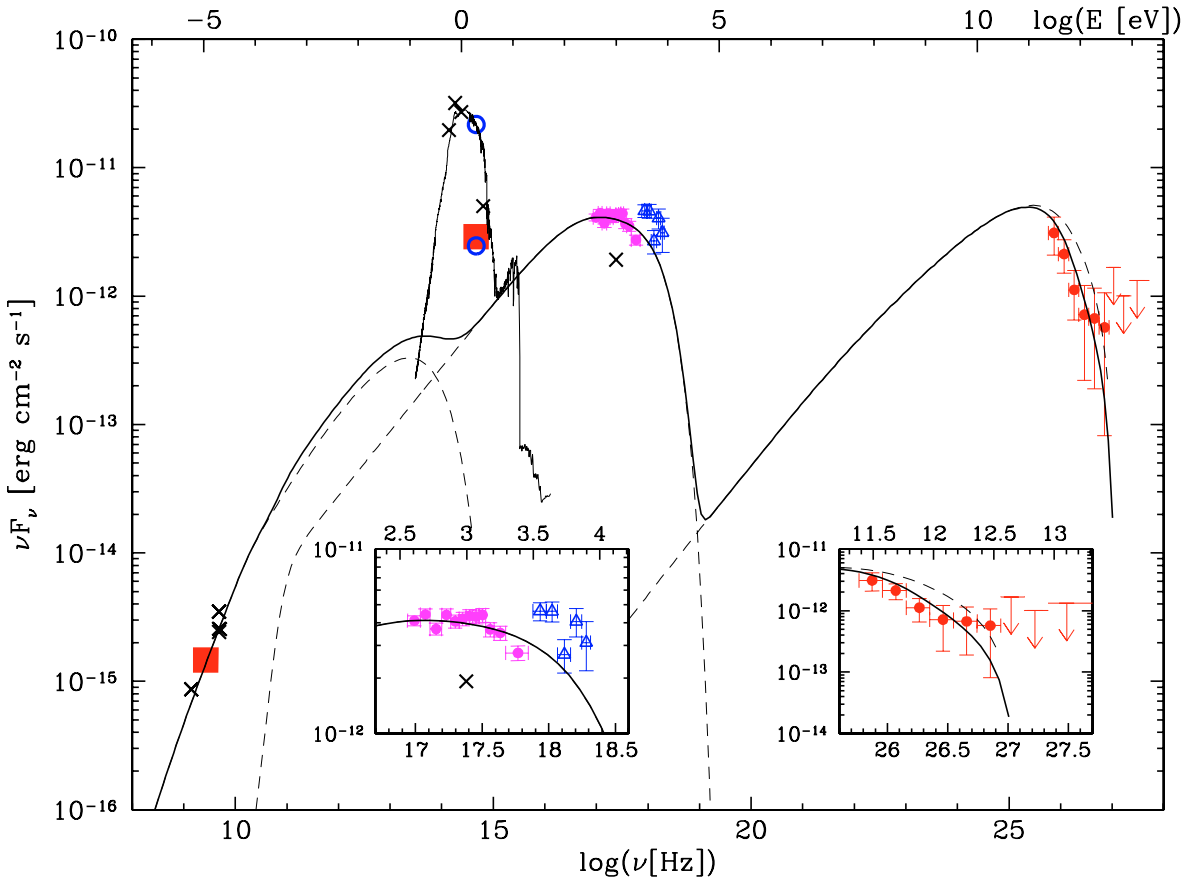

Fig. 4. The spectral energy distribution of RGB J0152+017. Shown are the HESS spectrum (red filled circles and upper limits), and contemporaneous RXTE (blue open triangles), Swift/XRT (corrected for Galactic absorption, magenta filled circles), optical host galaxysubtracted (ATOM) and radio (Nançay) observations (large red filled squares). The black crosses are archival data. The blue open points in the optical $R$-band correspond to the total and the core fluxes from Nilsson et al. (2003). A blob-in-jet synchrotron self-Compton model (see text) applied to RGB J0152+017 is also shown, describing the soft X-ray and VHE parts of the SED, with a simple synchrotron model shown at low frequencies to describe the extended part of the jet. The contribution of the dominating host galaxy is shown in the optical band. The dashed line above the solid line at VHE shows the source spectrum after correcting for EBL absorption. The left- and righthand side inlays detail portions of the observed $\mathrm{X}$-ray and VHE spectrum, respectively. or a central stellar population - so that it is considered as an upper limit in the following SSC model. A model including the optical ATOM data with possible additional contributions is beyond the scope of this paper.

We applied a non-thermal leptonic SSC model (Katarzyński et al. 2001) to account for the contemporaneous observations by Swift in X-rays and by HESS in the VHE band. The radio data are assumed to originate in an extended region, described by a separate synchrotron model for the extended jet (Katarzyński et al. 2001) to explain the low-frequency part of the SED (as in, e.g., Aharonian et al. 2005, 2008).

We should emphasise that the aim of applying this model in this work is not to present a definitive interpretation for this source, but rather to show that a standard SSC model is able to account for the VHE and Swift X-ray observations.

For the SSC model, we describe the system as a small homogeneous spherical, emitting region (blob) of radius $R$ within the jet, filled with a tangled magnetic field $B$ and propagating with a Doppler factor $\delta=[\Gamma(1-\beta \cos \theta)]^{-1}$. Here $\Gamma$ is the bulk Lorentz factor of the emitting plasma blob, $\beta=v / c$, and $\theta$ is the angle of the velocity vector, with respect to the line-of-sight. The electron energy distribution (EED) is described by a broken powerlaw, with indices $n_{1}$ and $n_{2}$, between Lorentz factors $\gamma_{\min }$ and $\gamma_{\max }$, with a break at $\gamma_{\text {break }}$ and density normalisation $K$.

The model also accounts for the absorption by the extragalactic background light (EBL) with the parameters given in Primack et al. (2005). RGB J0152+017 is too nearby $(z=0.08)$ to add to the constraints on the EBL that were found by HESS measurements of other blazars (Aharonian et al. 2006c). In all the models, we assume $H_{0}=70 \mathrm{~km} \mathrm{~s}^{-1} \mathrm{Mpc}^{-1}$, giving a luminosity distance of $d_{\mathrm{L}}=1.078 \times 10^{27} \mathrm{~cm}$ for RGB J0152+017.

The EED can be described by $K=3.1 \times 10^{4} \mathrm{~cm}^{-3}$, $\gamma_{\min }=1$, and $\gamma_{\max }=4 \times 10^{5}$. The break energy is assumed at $\gamma_{\text {break }}=7.0 \times 10^{4}$ and is consistent with the Swift/XRT spectrum, while providing good agreement with the HESS data. We assume the canonical index $n_{1}=2.0$ for the low-energy part of the EED, in accordance with standard Fermi-type acceleration mechanisms. The value $n_{2}=3.0$ for the high-energy part of the EED is constrained by the high-energy part of the X-ray spectrum. A good solution is found with the emitting region characterised by $\delta=25, R=1.5 \times 10^{15} \mathrm{~cm}$, and $B=0.10 \mathrm{G}$.

For the extended jet, the data are described well by $R_{\text {jet }}=$ $10^{16} \mathrm{~cm}, \delta_{\text {jet }}=7, K_{\text {jet }}=70 \mathrm{~cm}^{-3}, B_{\text {jet }}=0.05 \mathrm{G}$, and $\gamma_{\text {break, jet }}=$ $10^{4}$ at the base of the jet, and $L_{\mathrm{jet}}=50 \mathrm{pc}$ (all the parameters are detailed in Katarzyński et al. 2001).

Assuming additional contributions in the optical band, the multi-wavelength SED can thus be explained with a standard shock-acceleration process. The parameters derived from the model are similar to previous results for this type of source (see, e.g., Ghisellini et al. 2002).

From the current Nançay radio data and the Swift X-ray data, we obtain a broad-band spectral index $\alpha_{r x} \sim 0.56$ between the radio and the X-ray domains. The obtained SED, the corresponding location of the synchrotron peak, and the flux and shape of the Swift spectrum lead us to conclude that RGB J0152+017 can clearly be classified as an HBL object at the time of HESS observations.

\section{Conclusion}

The HBL RGB J0152+017 was detected in VHE at energies $>300 \mathrm{GeV}$ with the HESS experiment. The contemporaneous Swift, RXTE, Nançay, ATOM, and HESS data allow the multiwavelength SED for RGB J0152+017 to be derived for the first time, and to clearly confirm its HBL nature at the time of the HESS observations. In general, large variations of the VHE flux are expected in TeV blazars, making further monitoring of this source to detect high states of the VHE flux (flares) desirable.

Acknowledgements. The support of the Namibian authorities and of the University of Namibia in facilitating the construction and operation of HESS is gratefully acknowledged, as is the support by the German Ministry for Education and Research (BMBF), the Max Planck Society, the French Ministry for Research, the CNRS-IN2P3 and the Astroparticle Interdisciplinary Programme of the CNRS, the UK Science and Technology Facilities Council (STFC), the IPNP of the Charles University, the Polish Ministry of Science and Higher Education, the South African Department of Science and Technology and National Research Foundation, and by the University of Namibia. We appreciate the excellent work of the technical support staff in Berlin, Durham, Hamburg, Heidelberg, Palaiseau, Paris, Saclay, and in Namibia in the construction and operation of the equipment. 
This research made use of the NASA/IPAC Extragalactic Database (NED). The authors thank the RXTE team for their prompt response to our ToO request and the professional interactions that followed. The authors acknowledge the use of the publicly available Swift data, as well as the public HEASARC software packages. This work uses data obtained at the Nançay Radio Telescope. The authors also thank Dr. Mira Véron-Cetty for fruitful discussions.

\section{References}

Aharonian, F., Akhperjanian, A. G., Aye, K.-M., et al. (HESS Collaboration) 2004, Astropart. Phys., 22, 109

Aharonian, F., Akhperjanian, A. G., Bazer-Bachi, A. R., et al. (HESS Collaboration) 2005, A\&A, 442, 895

Aharonian, F., Akhperjanian, A. G., Bazer-Bachi, A. R., et al. (HESS Collaboration) 2006a, A\&A, 457, 899

Aharonian, F., Akhperjanian, A. G., Bazer-Bachi, A. R., et al. (HESS Collaboration) 2006b, A\&A, 448, L19

Aharonian, F., Akhperjanian, A. G., Bazer-Bachi, A. R., et al. (HESS Collaboration) 2006c, Nature, 440, 1018

Aharonian, F., Akhperjanian, A. G., Barres de Almeida, U., et al. (HESS Collaboration) 2008, A\&A, 477, 481

Becker, R. H., White, R. L., \& Edwards, A. L. 1991, ApJS, 75, 1

Brinkmann, W., Siebert, J., Feigelson, E. D., et al. 1997, A\&A, 323, 739

Burrows, D. N., Hill, J. E., Nousek, J. A., et al. 2005, Space Sci. Rev., 120, 165

Costamante, L., \& Ghisellini, G. 2002, A\&A, 384, 56

Feldman, G. J., \& Cousins, R. D. 1998, Phys. Rev. D, 57, 3873

Fioc, M., \& Rocca-Volmerange, B. 1997, A\&A, 326, 950

Ghisellini, G., Celotti, A., \& Costamante, L. 2002, A\&A, 386, 833

Griffith, M. R., Wright, A. E., Burke, B. F., \& Ekers, R. D. 1995, ApJS, 97, 347

Hauser, M., Möllenhoff, C., Pühlhofer, G., et al. 2004, Astron. Nachr., 325, 659

Hillas, A. M. 1985, Int. Cosmic Ray Conf., 3, 445

Jahoda, K., Swank, J. H., Giles, A. B., et al. 1996, Proc. SPIE, 2808, 59

Kalberla, P. M. W., Burton, W. B., Hartmann, D., et al. 2005, A\&A, 440, 775

Katarzyński, K., Sol, H., \& Kus, A. 2001, A\&A, 367, 809

Krawczynski, H., Coppi, P. S., Maccarone, T., \& Aharonian, F. A. 2000, A\&A, 353,97

Laurent-Muehleisen, S. A., Kollgaard, R. I., Ciardullo, R., et al. 1998, ApJS, 118,127

Laurent-Muehleisen, S. A., Kollgaard, R. I., Feigelson, E. D., Brinkmann, W., \& Siebert, J. 1999, ApJ, 525, 127

Li, T.-P., \& Ma, Y.-Q. 1983, ApJ, 272, 317

Nedbal, D., Benbow, W., Costamante, L., et al. 2007, The Astronomer's Telegram, 1295, 1

Nilsson, K., Pursimo, T., Heidt, J., et al. 2003, A\&A, 400, 95

Ott, M., Witzel, A., Quirrenbach, A., et al. 1994, A\&A, 284, 331

Primack, J. R., Bullock, J. S., \& Somerville, R. S. 2005, High Energy GammaRay Astron., 745, 23

Theureau, G., Hanski, M. O., Coudreau, N., Hallet, N., \& Martin, J.-M. 2007, A\&A, 465, 71

Young, P. J. 1976, AJ, 81, 807

\footnotetext{
1 Max-Planck-Institut für Kernphysik, Heidelberg, Germany

2 Yerevan Physics Institute, Yerevan, Armenia
}

${ }^{3}$ Centre d'Étude Spatiale des Rayonnements, CNRS/UPS, Toulouse, France

${ }^{4}$ Universität Hamburg, Institut für Experimentalphysik, Hamburg, Germany

5 Institut für Physik, Humboldt-Universität zu Berlin, Berlin, Germany

${ }^{6}$ LUTH, Observatoire de Paris, CNRS, Université Paris Diderot, Meudon, France

e-mail: jean-philippe.lenain@obspm. fr

7 IRFU/DSM/CEA, CE Saclay, Gif-sur-Yvette, France

8 University of Durham, Department of Physics, Durham, UK

9 Unit for Space Physics, North-West University, Potchefstroom, South Africa

${ }_{10}$ Laboratoire Leprince-Ringuet, École Polytechnique, CNRS/IN2P3, Palaiseau, France

${ }_{11}$ Laboratoire d'Annecy-le-Vieux de Physique des Particules, CNRS/IN2P3, Annecy-le-Vieux, France

12 Astroparticule et Cosmologie (APC), CNRS, Université Paris 7 Denis Diderot, Paris; UMR 7164 (CNRS, Université Paris 7, CEA, Observatoire de Paris), France

13 Dublin Institute for Advanced Studies, Dublin, Ireland

14 Landessternwarte, Universität Heidelberg, Heidelberg, Germany

15 Laboratoire de Physique Théorique et Astroparticules, CNRS/IN2P3, Université Montpellier II, Montpellier, France

${ }_{16}$ Universität Erlangen-Nürnberg, Physikalisches Institut, Erlangen, Germany

17 Laboratoire d'Astrophysique de Grenoble, INSU/CNRS, Université Joseph Fourier, Grenoble, France

18 Institut für Astronomie und Astrophysik, Universität Tübingen, Tübingen, Germany

19 LPNHE, Université Pierre et Marie Curie Paris 6, Université Denis Diderot Paris 7, CNRS/IN2P3, Paris, France

${ }^{20}$ Institute of Particle and Nuclear Physics, Charles University, Prague, Czech Republic

e-mail: dalibor.nedbal@mpi-hd.mpg.de

${ }^{21}$ Institut für Theoretische Physik, Lehrstuhl IV: Weltraum und Astrophysik, Ruhr-Universität Bochum, Bochum, Germany

22 University of Namibia, Windhoek, Namibia

${ }^{23}$ Obserwatorium Astronomiczne, Uniwersytet Jagielloński, Kraków, Poland

${ }^{24}$ Nicolaus Copernicus Astronomical Center, Warsaw, Poland

25 School of Physics \& Astronomy, University of Leeds, Leeds, UK

26 School of Chemistry \& Physics, University of Adelaide, Adelaide, Australia

27 Toruń Centre for Astronomy, Nicolaus Copernicus University, Toruń, Poland

28 European Associated Laboratory for Gamma-Ray Astronomy, jointly supported by CNRS and MPG 